\title{
Lexicographic Product and Isoperimetric Number
}

\author{
Ersin Aslan ${ }^{1}$ and Alpay Kirlangic ${ }^{2}$ \\ ${ }^{1}$ Turgutlu Vocational Training School, Celal Bayar University, Turgutlu-Manisa, Turkey \\ ${ }^{2}$ Department of Mathematics, Ege University, Izmir, Turkey \\ Correspondence should be addressed to Alpay Kirlangic; ersinaslan2000@gmail.com
}

Received 26 June 2013; Accepted 28 July 2013

Academic Editors: M. Caramia, P. E. Jorgensen, and S. Richter

Copyright (c) 2013 E. Aslan and A. Kirlangic. This is an open access article distributed under the Creative Commons Attribution License, which permits unrestricted use, distribution, and reproduction in any medium, provided the original work is properly cited.

The isoperimetric number of a graph $G$, denoted by $i(G)$, was introduced by Mohar (1987). A graph $G$ and a subset $X$ of its vertices are given, and let $\partial(X)$ denote the edge boundary of $X$, the set of edges which connects vertices in $X$ to vertices not in $X$. The isoperimetric number of $G$ is defined as $i(G)=\min _{1 \leq|X| \leq|V(G)| / 2}(|\partial(X)| /|X|)$. In this paper, some results about the isoperimetric number of graphs obtained by graph operations are given.

\section{Introduction}

Given a graph $G$ and a subset $X$ of its vertices, let $\partial(X)$ denote the edge boundary of $X$ that is, the set of edges which connects vertices in $X$ with vertices not in $X$. The isoperimetric number is defined as

$$
i(G)=\min _{1 \leq|X| \leq|V(G)| / 2} \frac{|\partial(X)|}{|X|} .
$$

Clearly, $i(G)$ can be defined in a more symmetric form by

$$
i(G)=\min \frac{|E(X, Y)|}{\min \{|X|,|Y|\}},
$$

where the minimum runs over all partitions of $V(G)=X \cup Y$ into nonempty subsets $X$ and $Y$, and $E(X, Y)=\partial X=\partial Y$ are the edges between $X$ and $Y$.

As examples of isoperimetric numbers, we consider the following.

(i) The isoperimetric number of the complete graph $K_{n}$ with $n$ vertices is $i\left(K_{n}\right)=\lceil n / 2\rceil$.

(ii) The isoperimetric number of the cycle $C_{n}$ with $n$ vertices is $i\left(C_{n}\right)=2 /\lfloor n / 2\rfloor$.

(iii) The isoperimetric number of the path $P_{n}$ with $n$ vertices is $i\left(P_{n}\right)=1 /\lfloor n / 2\rfloor$. (iv) The isoperimetric number of the complete bipartite graph with $m+n$ vertices $K_{m, n}$ is

$$
i\left(K_{m, n}\right)= \begin{cases}\frac{m n}{m+n}, & \text { if } m \text { and } n \text { are even } \\ \frac{m n+1}{m+n}, & \text { if } m \text { and } n \text { are odd } \\ \frac{m n}{m+n-1}, & \text { if } m+n \text { is odd. }\end{cases}
$$

It can be briefly shown as $i\left(K_{m, n}\right)=\lceil m n / 2\rceil /\lfloor(m+n) / 2\rfloor$.

The isoperimetric number is also closely related to the notion of bisection width, $b w(G)$, of a graph $G$. This is the minimum number of edges that must be removed from $G$ in order to split $V(G)$ into two equal-sized (within one if the number of vertices in $G$ is odd) subsets:

$$
b w(G)=\min _{|X|=\lfloor|V(G)| / 2\rfloor}|\partial X|,
$$

where $X \subset V(G)$. If known, one can use the isoperimetric number of a graph $G$ to establish a lower bound for its bisection width using the fact that

$$
\frac{b w(G)}{\lfloor|V(G)| / 2\rfloor} \geq i(G) .
$$

See [1]. 
The importance of $i(G)$ lies in various interesting interpretations of this number by Mohar as follows [2].

(a) From (2), it is evident that, in trying to determine $i(G)$, we have to find a small edge-cut $E(X, Y)$ separating as large a subset $X$ (assume $|X| \leq|Y|$ ) as possible from the remaining larger part $Y$. So, it is evident that $i(G)$ can serve as measure of connectivity of graphs. It seems that there might be possible applications in problems concerning connected networks, and the ways to "destroy" them are by removing a large portion of the network by cutting only a few edges.

(b) The problem of the partitioning $V(G)$ into two equally sized subsets (to within one element), in such a way that the number of the edges in the cut is minimal, is known as the bisectionwidth problem. It is important in VLSI design and some other practical applications. Clearly, it is related to isoperimetric number.

Theorem 1 (see [2]). Some of the theorems that Mohar stated are given below.

(a) $i(G)=0$ if and only if $G$ is disconnected.

(b) If $G$ is $k$-edge-connected then $i(G) \geq 2 k /|V(G)|$.

(c) If $\delta(G)$ is the minimal degree of vertices in $G$ then $i(G) \leq$ $\delta(G)$.

(d) If $e=u v$ is an edge of $G$ and $|V(G)| \geq 4$ then $i(G) \leq$ $[\operatorname{deg}(u)+\operatorname{deg}(v)-2] / 2$.

(e) If $\Delta$ is the maximum vertex degree in $G$ then $i(G) \leq$ $(\Delta-2)+2 /\lfloor|V(G)| / 2\rfloor$. If $G$ has a cycle with almost half the vertices of $G$ then $i(G) \leq \Delta-2$.

If a set $X \subset V(G)$ with $|X| \leq(1 / 2)|V(G)|$ reaches the minimum $i(G)=|\partial(X)| /|X|$ we call it an isoperimetric set. For $U \subseteq V(G)$ denoted by $G \mid U$, the subgraph of $G$ is induced on $U$ [2].

Proposition 2 (see [2]). If $G$ is a connected graph then it has an isoperimetric set $X$ such that $G \mid X$ and $G \mid(V \backslash X)$ are connected subgraphs of $G$.

In the next section, we prove a upper bound for isoperimetric number of lexicographic product of graphs.

\section{Lexicographic Product}

The lexicographic product $G_{1}\left[G_{2}\right]$ of two graphs $G_{1}$ and $G_{2}$ has its vertex set $V\left(G_{1}\right) \times V\left(G_{2}\right)$ with $\left(u_{1}, u_{2}\right)$ adjacent to $\left(v_{1}, v_{2}\right)$ if either $u_{1}$ adjacent to $v_{1}$ in $G_{1}$ or $u_{1}=v_{1}$ and $u_{2}$ are adjacent to $v_{2}$ in $G_{2}$. Note that unlike the union, join, and Cartesian product, this operation is not commutative.

Theorem 3. Let $G$ be a graph with $m$ vertices, and let $q$ edges and $H$ be a graph with $n$ vertices. Then,

$$
i(G[H]) \leq \min \left\{i(G) n, \frac{2 q\lceil n / 2\rceil}{m}+\frac{b w(H)}{\lfloor n / 2\rfloor}\right\} .
$$

Proof. Let $X_{G} \subseteq V(G)$ and $X_{G}$ be the isoperimetric set of $G$ and $\partial_{G}(X)$ edge boundary of $G$. We know that $G[H]$ includes $n$ copies of $G$. If $|X|=\left|X_{G}\right| n$ then $|\partial(X)| \leq\left|\partial_{G}(X)\right| n n$. Hence,

$$
\frac{|\partial(X)|}{|X|} \leq \frac{\left|\partial_{G}(X)\right| n n}{\left|X_{G}\right| n}=\frac{\left|\partial_{G}(X)\right| n n}{\left|X_{G}\right| n}=\frac{\left|\partial_{G}(X)\right| n}{\left|X_{G}\right|}
$$

So,

$$
\begin{aligned}
\min \left\{\frac{|\partial(X)|}{|X|}\right\} & \leq \min \left\{\frac{\left|\partial_{G}(X)\right| n}{\left|X_{G}\right|}\right\} \\
& =\min \left\{\frac{\left|\partial_{G}(X)\right|}{\left|X_{G}\right|}\right\} n=i(G) n .
\end{aligned}
$$

Similarly, let $X_{H} \subseteq V(H)$ with $\left|X_{H}\right| \leq\lfloor n / 2\rfloor$ and $\left|X_{H}\right|=r m$. We know that $G[H]$ includes $m$ copies of $H$. If $|X|=r m$ then we have $|\partial(X)| \leq r(n-r) 2 q+\left|\partial_{H}(X)\right| m$. Therefore, $|\partial(X)| /|X| \leq\left(r(n-r) 2 q+\left|\partial_{H}(X)\right| m\right) / r m$. So, $\min \{|\partial(X)| /|X|\} \leq \min \left\{\left(r(n-r) 2 q+\left|\partial_{H}(X)\right| m\right) / r m\right\}$. The function $\left(r(n-r) 2 q+\left|\partial_{H}(X)\right| m\right) / r m$ takes its minimum value at $r=\lfloor n / 2\rfloor$. Since $\left|X_{H}\right|=\lfloor n / 2\rfloor$ then $|\partial(X)|=b w(H)$. We have

$$
i(G[H]) \leq \frac{2 q\lceil n / 2\rceil}{m}+\frac{b w(H)}{\lfloor n / 2\rfloor} .
$$

The proof is completed by (8) and (9).

We have $i(G) n \leq 2 q\lceil n / 2\rceil) / m+b w(H) /\lfloor n / 2\rfloor$ according to the upper bounds of $i(G)$, and hence we get $i(G[H]) \leq i(G) n$.

Corollary 4. Let $G$ be a graph with $m$ vertices, and let $q$ edges and $H$ be a graph with $n$ vertices. If $n$ is even and $i(G) \leq q / m$ and $i(G) \leq 2 b w(H) n^{2}$ then

$$
i(G) n \leq \frac{2 q\lceil n / 2\rceil}{m}+\frac{b w(H)}{\lfloor n / 2\rfloor} .
$$

Proof. If $n$ is even and $i(G) \leq 2 b w(H) / n^{2}$ then $i(G) n^{2} \leq$ $2 b w(H)$. Hence, we have $i(G) n^{2}+q n^{2} \leq 2 b w(H)+q n^{2}$. Since $i(G) \leq q / m$ then $n^{2}(i(G)+i(G) m) \leq n^{2}(i(G)+q) \leq$ $i(G) n^{2}+q n^{2} \leq 2 b w(H)+q n^{2}$. So, we have $i(G) m n^{2} \leq$ $n^{2}(i(G)+i(G) m) \leq 2 b w(H)+q n^{2}$. Therefore, we have

$$
\begin{aligned}
\frac{i(G) m n^{2}}{m n} \leq \frac{2 b w(H)+q n^{2}}{m n} & =\frac{2 q(n / 2)}{m}+\frac{b w(H)}{n / 2} \\
& =\frac{2 q\lceil n / 2\rceil}{m}+\frac{b w(H)}{\lfloor n / 2\rfloor} .
\end{aligned}
$$

Corollary 5. Let $G$ be a graph with $m$ vertices, and let $q$ edges and $H$ be a graph with $n$ vertices. If $n$ is odd and $i(G) \leq$ $2 b w(H) n^{2}-n$ then

$$
i(G) n \leq \frac{2 q\lceil n / 2\rceil}{m}+\frac{b w(H)}{\lfloor n / 2\rfloor} .
$$

Corollary 6. Let $T$ be a tree with $m$ vertices, and let $H$ be a graph with $n$ vertices. If $n$ is even then

$$
i(T) n \leq \frac{2(m-1)\lceil n / 2\rceil}{m}+\frac{b w(H)}{\lfloor n / 2\rfloor} .
$$


Proof. For $n>1$ and $m>1$, we know that $0 \leq(m-2) n^{2}+2 m$. Then, $m n^{2} \leq(2 m-2) n^{2}+2 m$. Since $b w(H) \geq 1$ and $i(T)<1$ then we have $i(T) m n^{2} \leq m n^{2} \leq(2 m-2) n^{2}+2 m b w(H)$. Therefore, we have

$$
\begin{aligned}
i(T) n & \leq \frac{2(m-1)(n / 2)}{m}+\frac{b w(H)}{n / 2} \\
& =\frac{2(m-1)\lceil n / 2\rceil}{m}+\frac{b w(H)}{\lfloor n / 2\rfloor} .
\end{aligned}
$$

Corollary 7. Let $T$ be a tree with $m$ vertices, and let $H$ be a graph with $n$ vertices. If $n$ is odd and $n-1<m$ then $i(T) n \leq$ $2(m-1)\lceil n / 2\rceil / m+b w(H) /\lfloor n / 2\rfloor$.

Corollary 8. Let $G$ be a graph with $m$ vertices, $q$ edges that is not a tree, and let $P_{n}$ be a path graph with $n$ vertices. If $n$ is even and $i(G) \leq(m-1) / m$ then

$$
i(G) n \leq \frac{2 q(n / 2)}{m}+\frac{1}{(n / 2)}=\frac{2 q\lceil n / 2\rceil}{m}+\frac{b w\left(P_{n}\right)}{\lfloor n / 2\rfloor} .
$$

Proof. If $n$ is even and $i(G) \leq(m-1) / m$ then $i(G) m \leq m-1$. Thus, $i(G) m n^{2} \leq(m-1) n^{2} \leq(m-1) n^{2}+2 m$. Therefore, $i(G) m n^{2} / m n \leq\left((m-1) n^{2}+2 m\right) / m n=(m-1) n / m+2 / n$. So,

$$
\begin{aligned}
i(G) n \leq \frac{(m-1) n}{m}+\frac{2}{n}= & \frac{2(m-1) n / 2}{m} \\
& +\frac{1}{n / 2} \leq \frac{2 q(n / 2)}{m}+\frac{1}{n / 2} \\
= & \frac{2 q\lceil n / 2\rceil}{m}+\frac{b w\left(P_{n}\right)}{\lfloor n / 2\rfloor} .
\end{aligned}
$$

Corollary 9. Let $G$ be a graph with $m$ vertices, q edges that is not a tree, and let $P_{n}$ be a path graph with $n$ vertices. If $n$ is odd and $i(G) \leq 2 /\left(n^{2}-n\right)$ then

$$
i(G) n \leq \frac{2 q((n+1) / 2)}{m}+\frac{1}{(n-1) / 2}=\frac{2 q\lceil n / 2\rceil}{m}+\frac{b w\left(P_{n}\right)}{\lfloor n / 2\rfloor} .
$$

Corollary 10. Let $G$ be a graph with $m$ vertices, $q$ edges that is not a tree, and let $C_{n}$ be a cycle graph with $n$ vertices. If $n$ is even and $i(G) \leq\left(n^{2}+4\right) / n^{2}$ then

$$
i(G) n \leq \frac{2 q(n / 2)}{m}+\frac{2}{n / 2}=\frac{2 q\lceil n / 2\rceil}{m}+\frac{b w\left(C_{n}\right)}{\lfloor n / 2\rfloor} .
$$

Proof. If $n$ is even and $i(G) \leq\left(n^{2}+4\right) / n^{2}$ then

$$
\begin{aligned}
i(G) n & \leq \frac{n^{2}+4}{n}=\frac{n^{2}}{n}+\frac{4}{n}=\frac{m n}{m}+\frac{2}{n / 2} \\
& =\frac{2 m n / 2}{m}+\frac{2}{n / 2} \leq \frac{2 q(n / 2)}{m}+\frac{2}{n / 2} .
\end{aligned}
$$

So,

$$
i(G) n \leq \frac{2 q(n / 2)}{m}+\frac{2}{n / 2}=\frac{2 q\lceil n / 2\rceil}{m}+\frac{b w\left(C_{n}\right)}{\lfloor n / 2\rfloor} .
$$

Corollary 11. Let $G$ be a graph with $m$ vertices, $q$ edges that is not a tree, and let $C_{n}$ be a cycle graph with $n$ vertices. If $n$ is odd and $i(G) \leq\left(n^{2}+3\right) /\left(n^{2}-n\right)$ then

$i(G) n \leq \frac{2 q((n+1) / 2)}{m}+\frac{2}{(n-1) / 2}=\frac{2 q\lceil n / 2\rceil}{m}+\frac{b w\left(C_{n}\right)}{\lfloor n / 2\rfloor}$.

Corollary 12. Let $G$ be a graph with $m$ vertices, q edges that is not a tree and let $K_{n}$ be a complete graph with $n$ vertices.

(a) If $n$ is even and $i(G) \leq(q / m)+(1 / 2)$ then

$$
i(G) n \leq \frac{2 q(n / 2)}{m}+\frac{n^{2} / 4}{n / 2}=\frac{2 q\lceil n / 2\rceil}{m}+\frac{b w\left(K_{n}\right)}{\lfloor n / 2\rfloor} .
$$

(b) If $n$ is odd and $i(G) \leq(n+1)(2 q+m) / m n$ then

$$
i(G) n \leq \frac{2 q\lceil n / 2\rceil}{m}+\frac{b w\left(K_{n}\right)}{\lfloor n / 2\rfloor} .
$$

Proof. (a) If $n$ is even and $i(G) \leq(q / m)+(1 / 2)$ then we have

$$
\begin{aligned}
i(G) n & \leq \frac{q n}{m}+\frac{n}{2} \\
& =\frac{2 q(n / 2)}{m}+\frac{n^{2} / 4}{n / 2} \\
& =\frac{2 q\lceil n / 2\rceil}{m}+\frac{b w\left(K_{n}\right)}{\lfloor n / 2\rfloor} .
\end{aligned}
$$

The proof for $n$ even is very similar to the proof for odd.

Theorem 13. Let $P_{m}$ be a path graph with $m$ vertices, and let $q$ edges and $H$ be a graph with $n$ vertices. Then,

$$
\begin{aligned}
& i\left(P_{m}[H]\right) \\
& = \begin{cases}\frac{2 n}{m}, & \text { if } m \text { is even } \\
\frac{2 n^{2}+2 b w(H)}{m n}, & \text { if } m \text { is odd and } n \text { is even } \\
\frac{2 n}{m-1}, & \text { and } b w(H)(m-1)<n^{2} \\
\frac{2 n^{2}+2 b w(H)}{m n-1}, & \text { if } m \text { is odd and } n \text { is even } m \text { and } n \text { are odd } \\
\frac{2 n}{m-1}, & \text { and bw }(H)(m-1)<n^{2}-n \\
& \text { if } m \text { and } n \text { are even }\end{cases} \\
& \text { and bw }(H)(m-1) \geq n^{2}-n .
\end{aligned}
$$

Proof. Let $V\left(P_{m}\right)=\{1,2, \ldots, m\}$ and $X \subseteq V\left(P_{m}[H]\right)$ with $|X| \leq\lfloor m n / 2\rfloor$. For $i=1,2, \ldots, m$ let $X_{i}=X \cap(V(H) \times$ i). Hence, $X$ is the disjoint union of $X_{1}, X_{2}, \ldots, X_{m}$. Let $S_{0}=\left\{X_{i}|| X_{i} \mid=0,1 \leq i \leq m\right\}$ and $S_{n}=\left\{X_{i}|| X_{i} \mid=\right.$ $n, 1 \leq i \leq m\}$. To prove this theorem, we have three cases. 
Case 1. Let $m$ be an even integer. To prove this case, we have three subcases.

Subcase 1.1. If $|X|=r$ where $1 \leq r<n$ then $|\partial(X)| \geq r n+1$. Therefore, $|\partial(X)| /|X| \geq(r n+1) / r$. The function $(r n+1) / r$ has its minimum value at $r=n-1$, and we have

$$
\min \left\{\frac{|\partial(X)|}{|X|}\right\} \geq \frac{(n-1) n+1}{n-1} .
$$

Subcase 1.2. If $S_{0}>0$ and $S_{n}>0$ and $|X|=r$ where $n \leq r \leq$ $m n / 2$ then $|\partial(X)| \geq n^{2}+\left(m-S_{0}-S_{n}\right)\left|\partial_{H}(X)\right|$. Therefore, $|\partial(X)| /|X| \geq\left(n^{2}+\left(m-S_{0}-S_{n}\right)\left|\partial_{H}(X)\right|\right) / r$. The function $\left(n^{2}+\left(m-S_{0}-S_{n}\right)\left|\partial_{H}(X)\right|\right) / r$ has its minimum value at $r=$ $m n / 2$. If $r=m n / 2$ then $\left(m-S_{0}-S_{n}\right)\left|\partial_{H}(X)\right|=0$. Thus,

$$
\min \left\{\frac{|\partial(X)|}{|X|}\right\} \geq \frac{2 n}{m}
$$

Subcase 1.3. If $\left(S_{0}=0, S_{n}=0\right)$ or $\left(S_{0}=0, S_{n}>0\right)$ or $\left(S_{0}>\right.$ $\left.0, S_{n}=0\right)$ and $|X|=r$ where $n \leq r \leq m n / 2$ then

$$
\begin{aligned}
|\partial(X)| \geq & \left\lfloor\frac{r}{m}\right\rfloor\left(n-\left\lfloor\frac{r}{m}\right\rfloor\right) 2 q+\left|\partial_{H}(X)\right| m \\
\geq & \left\lfloor\frac{r}{m}\right\rfloor\left(n-\left\lfloor\frac{r}{m}\right\rfloor\right) 2(m-1) \\
& +\left|\partial_{H}(X)\right| m \geq\left\lfloor\frac{r}{m}\right\rfloor\left(n-\left\lfloor\frac{r}{m}\right\rfloor\right)(2 m-2)+m .
\end{aligned}
$$

Thus, $|\partial(X)| /|X| \geq(\lfloor r / m\rfloor(n-\lfloor r / m\rfloor)(2 m-2)+m) / r$. The function $(\lfloor r / m\rfloor(n-\lfloor r / m\rfloor)(2 m-2)+m) / r$ has its minimum value at $r=m n / 2$, and we have

$$
\begin{aligned}
\min & \left\{\frac{|\partial(X)|}{|X|}\right\} \\
& \geq \frac{\lfloor(m n / 2) / m\rfloor(n-\lfloor(m n / 2) / m\rfloor)(2 m-2)+m}{m n / 2} \\
& =\frac{n^{2} m-n^{2}+2 m}{m n} .
\end{aligned}
$$
$(2 n / m)$.

By (26), (27), and (29), if $m$ is even then $i\left(P_{m}[H]\right)=$

Case 2. Let $m$ be an odd, and let $n$ be an even integer. To prove this case, we have four subcases.

Subcase 2.1. If $|X|=r$ where $1 \leq r<n$ then $|\partial(X)| \geq r n+1$. Therefore, $|\partial(X)| /|X| \geq(r n+1) / r$. The function $(r n+1) / r$ has its minimum value at $r=n-1$, and we have

$$
\min \left\{\frac{|\partial(X)|}{|X|}\right\} \geq \frac{(n-1) n+1}{n-1} .
$$

Subcase 2.2. If $S_{0}>0$ and $S_{n}>0$ and $|X|=r$ where $n \leq$ $r \leq(m-1) n / 2$ then $|\partial(X)| \geq n^{2}+\left(m-S_{0}-S_{n}\right)\left|\partial_{H}(X)\right|$. Therefore, $|\partial(X)| /|X| \geq\left(n^{2}+\left(m-S_{0}-S_{n}\right)\left|\partial_{H}(X)\right|\right) / r$. The function $\left(n^{2}+\left(m-S_{0}-S_{n}\right)\left|\partial_{H}(X)\right|\right) / r$ has its minimum value at $r=(m-1) n / 2$. If $r=(m-1) n / 2$ then $\left(m-S_{0}-S_{n}\right)\left|\partial_{H}(X)\right|=$ 0 . Thus,

$$
\min \left\{\frac{|\partial(X)|}{|X|}\right\} \geq \frac{n^{2}}{(m-1) n / 2}=\frac{2 n}{m-1} .
$$

Subcase 2.3. If $S_{0}>0$ and $S_{n}>0$ and $|X|=r$ where $(m-$ 1) $n / 2 \leq r \leq m n / 2$ then $|\partial(X)|=n^{2}+b w(H)$. Therefore, $|\partial(X)| /|X|=\left(n^{2}+b w(H)\right) / r$. The function $\left(n^{2}+b w(H)\right) / r$ has its minimum value at $r=m n / 2$. Thus,

$$
\min \left\{\frac{|\partial(X)|}{|X|}\right\}=\frac{n^{2}+b w(H)}{m n / 2}=\frac{2 n^{2}+2 b w(H)}{m n} .
$$

Subcase 2.4. If $\left(S_{0}=0, S_{n}=0\right)$ or $\left(S_{0}=0, S_{n}>0\right)$ or $\left(S_{0}>\right.$ $\left.0, S_{n}=0\right)$ and $|X|=r$ where $n \leq r \leq m n / 2$ then

$$
\begin{aligned}
|\partial(X)| & \geq\left\lfloor\frac{r}{m}\right\rfloor\left(n-\left\lfloor\frac{r}{m}\right\rfloor\right) 2 q+\left|\partial_{H}(X)\right| m \\
& \geq\left\lfloor\frac{r}{m}\right\rfloor\left(n-\left\lfloor\frac{r}{m}\right\rfloor\right) 2(m-1)+\left|\partial_{H}(X)\right| m \\
& \geq\left\lfloor\frac{r}{m}\right\rfloor\left(n-\left\lfloor\frac{r}{m}\right\rfloor\right)(2 m-2)+m .
\end{aligned}
$$

Thus, $|\partial(X)| /|X| \geq(\lfloor r / m\rfloor(n-\lfloor r / m\rfloor)(2 m-2)+m) / r$. The function $(\lfloor r / m\rfloor(n-\lfloor r / m\rfloor)(2 m-2)+m) / r$ takes minimum value at $r=m n / 2$, and we have

$$
\begin{aligned}
\min & \left\{\frac{|\partial(X)|}{|X|}\right\} \\
\geq & \frac{\lfloor(m n / 2) / m\rfloor(n-\lfloor(m n / 2) / m\rfloor)(2 m-2)+m}{m n / 2} \\
& =\frac{n^{2} m-n^{2}+2 m}{m n} .
\end{aligned}
$$

By (30), (31), (32), and (34), we have that if $m$ is odd and $n$ is even and $b w(H)(m-1)<n^{2}$ then $i\left(P_{m}[H]\right)=\left(2 n^{2}+\right.$ $2 b w(H)) / m n$, and if $m$ is odd and $n$ is even and $b w(H)(m-$ 1) $\geq n^{2}$ then $i\left(P_{m}[H]\right)=2 n /(m-1)$.

Case 3. The proofs of the case in which $m$ and $n$ are odd are similar to that of Case 2.

The isoperimetric number of $i\left(P_{m}\left[P_{n}\right]\right)$ is given in the following corollary.

Corollary 14. Let $m$ and $n$ be positive integers. Then,

$$
\begin{aligned}
& i\left(P_{m}\left[P_{n}\right]\right) \\
& = \begin{cases}\frac{2 n}{m}, & \text { if } m \text { is even } \\
\frac{2 n^{2}+2}{m n}, & \text { if } m \text { is odd and } n \text { is even } \\
\frac{2 n}{m-1}, & \text { and }(m-1)<n^{2} \\
\frac{2 n^{2}+2}{m n-1}, & \text { if } m \text { is odd and } n \text { is even } \text { odd and } n \text { is odd } \\
\frac{2 n}{m-1}, & \text { if } m \text { is odd and } n \text { is even }\end{cases}
\end{aligned}
$$


The isoperimetric number of $i\left(P_{m}\left[C_{n}\right]\right)$ is given in the following corollary.

Corollary 15. Let $m>5$ and $n$ be positive integers. Then,

$$
\begin{aligned}
& i\left(P_{m}\left[C_{n}\right]\right) \\
& = \begin{cases}\frac{2 n}{m}, & \text { if } m \text { is even } \\
\frac{2 n^{2}+4}{m n}, & \text { if } m \text { is odd and } n \text { is even } \\
\frac{2 n}{m-1}, & \text { and } 2(m-1)<n^{2} \\
\frac{2 n^{2}+4}{m n-1}, & \text { if } m \text { is odd and } n \text { is even } \\
\frac{2 n}{m-1}, & \text { if } m \text { is odd and } n \text { is odd } n \text { is even }\end{cases}
\end{aligned}
$$

The isoperimetric number of $i\left(C_{m}\left[P_{n}\right]\right)$ is given in the following corollary.

Corollary 16. Let $m>5$ and $n$ be positive integers. Then,

$$
\begin{aligned}
& i\left(C_{m}\left[P_{n}\right]\right) \\
& = \begin{cases}\frac{4 n}{m}, & \text { if } m \text { and } n \text { are even } \\
\frac{4 n^{2}+2}{m n}, & \text { if } m \text { is odd and } n \text { is even } \\
\frac{4 n}{n^{2}+1}, & \text { and } m \leq 2 n^{2}+1 \\
\frac{n^{2}-1}{n}, & \text { if } m \text { is odd and } n \text { is even } m \text { is even and } n \text { is odd } \\
\frac{4 n}{m}, & \text { and } m \leq \frac{4 n^{2}}{n^{2}-1} \\
\frac{4 n^{2}}{m-1) n}, & \text { if } m \text { is even and } n \text { is odd } \text { odd and } n \text { is odd } \\
\frac{4 n^{2}+2}{m n-1}, & \text { and } m \leq 2 n^{2}-2 n+1 \\
\text { if odd and } n \text { is odd } m>\frac{4 n^{2}}{n^{2}-1}\end{cases}
\end{aligned}
$$

The isoperimetric number of $i\left(K_{m}\left[P_{n}\right]\right)$ is given in the following corollary.
Corollary 17. Let $m$ and $n$ be positive integers. Then,

$$
\begin{aligned}
& i\left(K_{m}\left[P_{n}\right]\right) \\
& = \begin{cases}\frac{n^{2} m-n^{2}+4}{2 n}, & \text { if } n \text { is even } \\
\frac{n^{2} m^{2}-n^{2} m+3 m}{2 m n}, & \text { if } m \text { is even and } n \text { is odd } \\
\frac{n^{2} m^{2}-n^{2} m+5 m-1}{2(m n-1)}, & \text { if } m \text { and } n \text { are odd. }\end{cases}
\end{aligned}
$$

The isoperimetric number of $i\left(K_{1, m}\left[P_{n}\right]\right)$ is given in the following corollary.

Corollary 18. Let $m$ and $n$ be positive integers. Then,

$$
\begin{aligned}
& i\left(K_{1, m}\left[P_{n}\right]\right)
\end{aligned}
$$

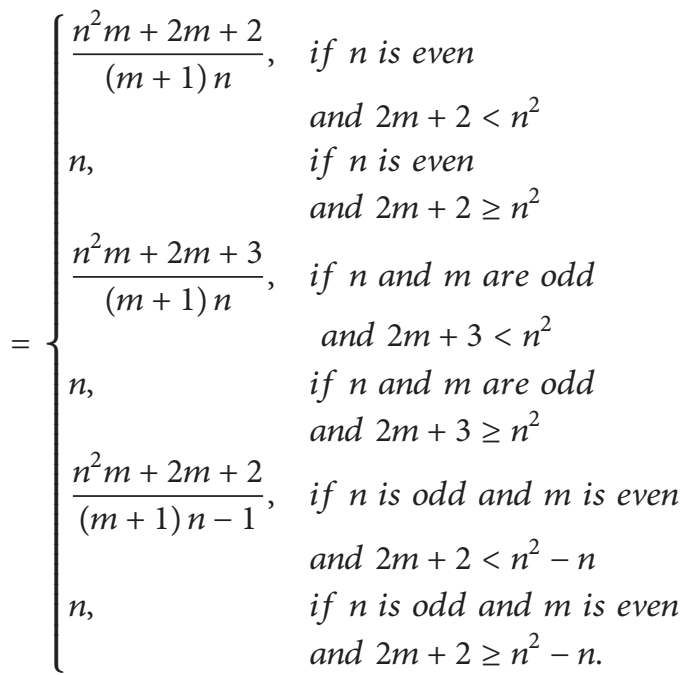

The isoperimetric number of $i\left(K_{1, m}\left[C_{n}\right]\right)$ is given in the following corollary.

Corollary 19. Let $m$ and $n$ be positive integers. Then,

$$
\begin{aligned}
& i\left(K_{1, m}\left[C_{n}\right]\right) \\
& = \begin{cases}\frac{n^{2} m+4 m+4}{(m+1) n}, & \text { if } n \text { is even } \\
n, & \begin{array}{l}
\text { and } 4 m+4<n^{2} \\
\text { if } n \text { is even }
\end{array} \\
\frac{n^{2} m+4 m+5}{(m+1) n}, & \text { and } 4 m+4 \geq n^{2} \\
n, & \text { if } n \text { and } m \text { are odd } 4 m+5<n^{2} \\
\frac{n^{2} m+4 m+4}{(m+1) n-1}, & \text { if } n \text { is odd and } m \text { is even } m \text { are odd } \\
n, & \text { and } 4 m+4<n^{2}-n \\
\text { if } n \text { is odd and } m \text { is even }\end{cases}
\end{aligned}
$$




\section{References}

[1] M. C. Azizoğlu and Ö. Eğecioğlu, "The isoperimetric number and the bisection width of generalized cylinders," in The Ninth Quadrennial International Conference on Graph Theory, Combinatorics, Algorithms and Applications, vol. 11 of Electronic Notes in Discrete Mathematics, pp. 1-10, Elsevier, Amsterdam, The Netherlands, 2002.

[2] B. Mohar, "Isoperimetric numbers of graphs," Journal of Combinatorial Theory. Series B, vol. 47, no. 3, pp. 274-291, 1989. 


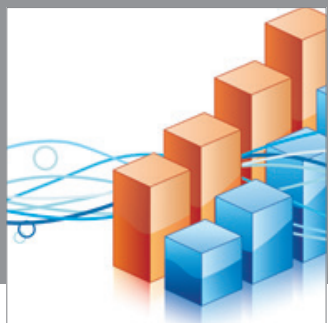

Advances in

Operations Research

mansans

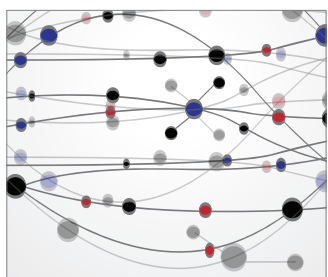

The Scientific World Journal
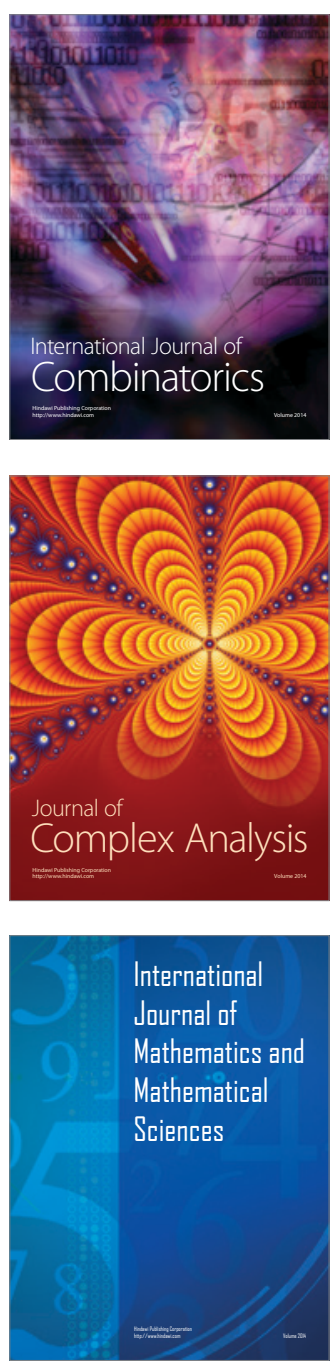
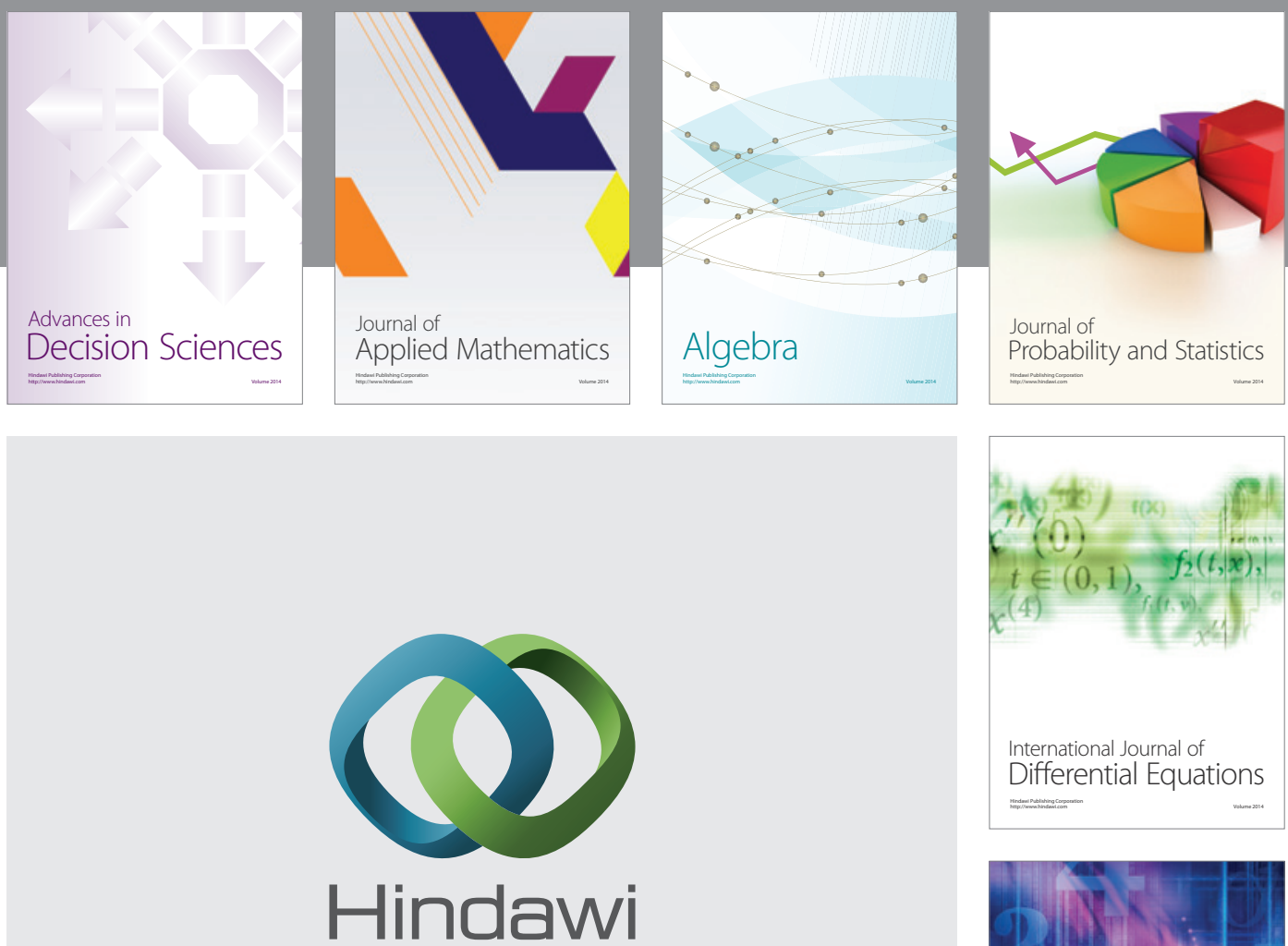

Submit your manuscripts at http://www.hindawi.com
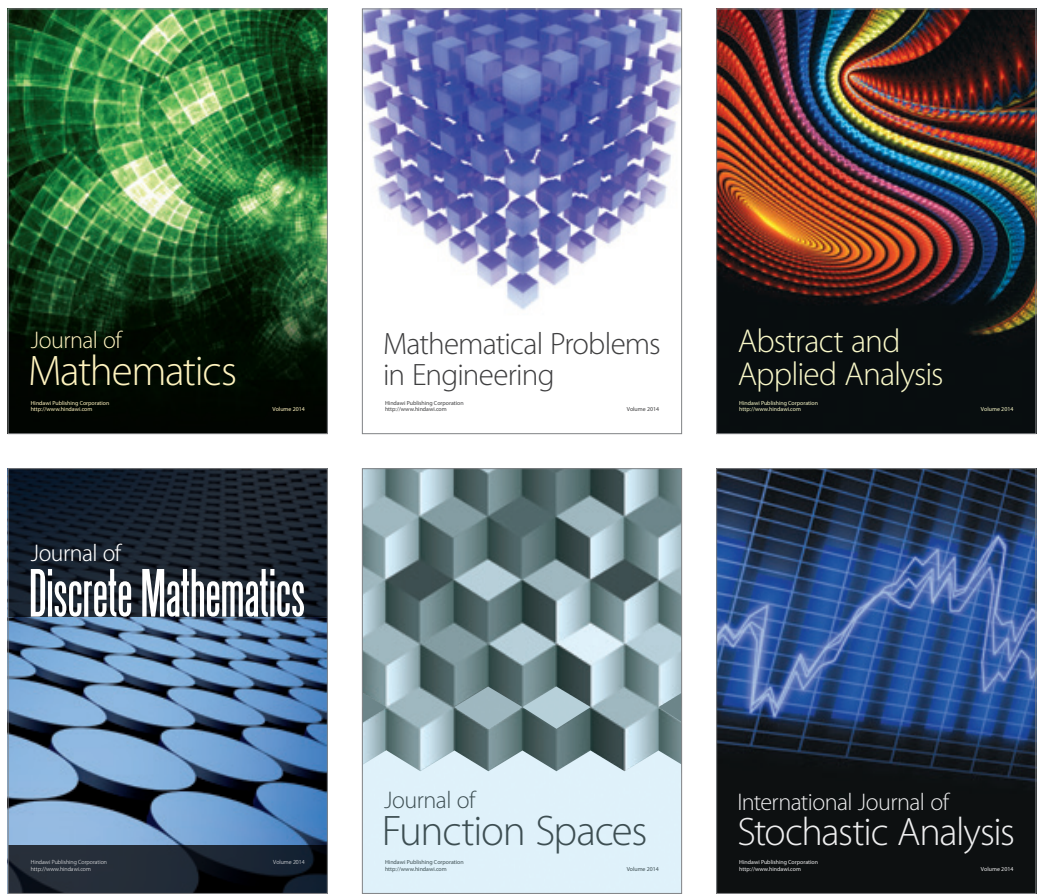

Journal of

Function Spaces

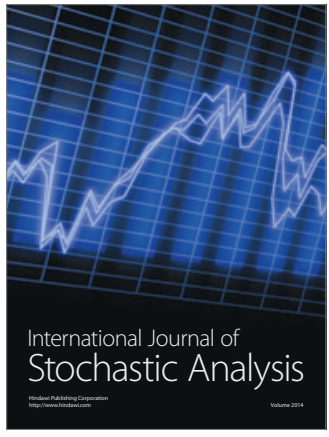

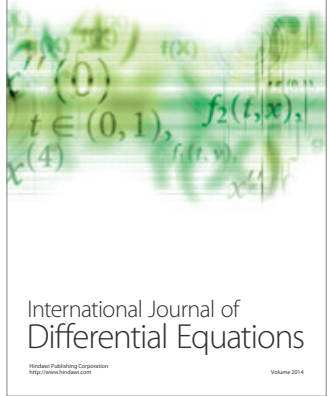
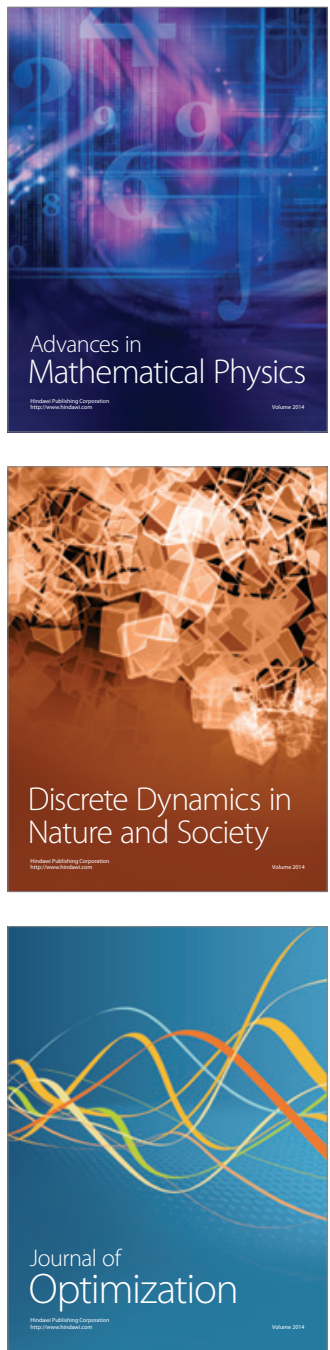